CRYSTALLOGRAPHIC COMMUNICATIONS

ISSN 2056-9890

Reçu le 6 août 2015

Accepté le 23 août 2015

Edité par H. Stoeckli-Evans, Université de Neuchâtel, Suisse

Keywords: crystal structure; cobalt complex; imidazole; hydrogen bonding; framework

CCDC reference: 1420121

Supporting information: this article has supporting information at journals.iucr.org/e



OPEN $\odot$ ACCESS

\section{Etude structurale et vibrationnelle d'un nouveau composé complexe de cobalt: $\left[\mathrm{Co}(\text { imidazole })_{4} \mathrm{Cl}\right] \mathrm{Cl}$}

\author{
Amira Derbel, ${ }^{a}$ Tahar Mhiri ${ }^{\mathrm{a}}$ et Mohsen Graia ${ }^{\mathrm{b} *}$ \\ aLaboratoire de l'état solide, Faculté des Sciences, Université de Sfax, BP W 3038 Sfax, Tunisie, et ${ }^{\mathbf{b}}$ Laboratoire de \\ Matériaux et Cristallochimie, Faculté des Sciences de Tunis, Université de Tunis El Manar, 2092 El Manar Tunis, Tunisie. \\ *Correspondence e-mail: mohseng2002@yahoo.fr
}

In the title complex, chloridotetrakis $\left(1 H\right.$-imidazole $\left.-\kappa N^{3}\right)$ cobalt(II) chloride, $\left[\mathrm{CoCl}\left(\mathrm{C}_{3} \mathrm{H}_{4} \mathrm{~N}_{2}\right)_{4}\right] \mathrm{Cl}$, the $\mathrm{Co}^{\mathrm{II}}$ cation has a distorted square-pyramidal coordination environment. It is coordinated by four $\mathrm{N}$ atoms of four imidazole (Im) groups in the basal plane, and by a $\mathrm{Cl}$ atom in the apical position. It is isostructural with $\left[\mathrm{Cu}(\mathrm{Im})_{4} \mathrm{Cl}\right] \mathrm{Cl}$ [Morzyk-Ociepa et al. (2012). J. Mol. Struct. 1028, 49-56] and $\left[\mathrm{Cu}(\mathrm{Im})_{4} \mathrm{Br}\right] \mathrm{Br}$ [Hossaini Sadr et al. (2004). Acta Cryst. E60, m1324-m1326]. In the crystal, the $\left[\mathrm{CoCl}\left(\mathrm{C}_{3} \mathrm{H}_{4} \mathrm{~N}_{2}\right)_{4}\right]^{+}$cations and $\mathrm{Cl}^{-}$anions are linked via $\mathrm{N}-\mathrm{H} \cdots \mathrm{Cl}$ hydrogen bonds, forming layers parallel to (010). These layers are linked via $\mathrm{C}-\mathrm{H} \cdots \mathrm{Cl}$ hydrogen bonds and $\mathrm{C}-\mathrm{H} \cdots \pi$ and $\pi-\pi$ [intercentroid distance $=3.794(2) \AA]$ interactions, forming a three-dimensional framework. The IR spectrum shows vibrational bands typical for imidazol groups. The monoclinic unit cell of the title compound emulates an orthorhombic cell as its $\beta$ angle is close to $90^{\circ}$. The crystal is twinned, with the refined ratio of twin components being 0.569 (1):0.431 (1).

\section{Contexte chimique}

L'imidazole et ses dérivés sont considérés parmi les hétérocycles les plus intéressants qui peuvent participer à la formation d'ions complexes. Ils se retrouvent couramment dans plusieurs molécules naturelles telles l'acide aminé histidine, la caféine, les purines ou la vitamine B12. En biologie, il s'agit d'un pharmacophore dont la toxicité est plutôt faible (OECD, 2003). Ainsi, le motif imidazole figure dans la formulation de nombreux médicaments, notamment des anti-inflammatoires, antifongiques, antihypertenseurs et même anticancéreux (Shargel et al., 2006; Castaño et al., 2008; Bogle et al., 1994).

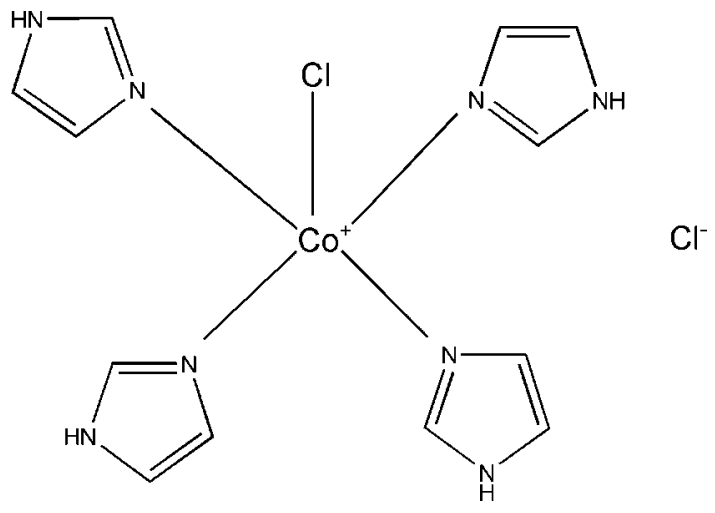

\section{Commentaire structurelle}

L'unité asymétrique du composé étudié $\left[\mathrm{Co}\left(\mathrm{C}_{3} \mathrm{H}_{4} \mathrm{~N}_{2}\right) \mathrm{Cl}\right] \mathrm{Cl}$ est formée d'un cation complexe $\left[\mathrm{Co}\left(\mathrm{C}_{3} \mathrm{H}_{4} \mathrm{~N}_{2}\right) \mathrm{Cl}\right]^{+}$et d'un anion 


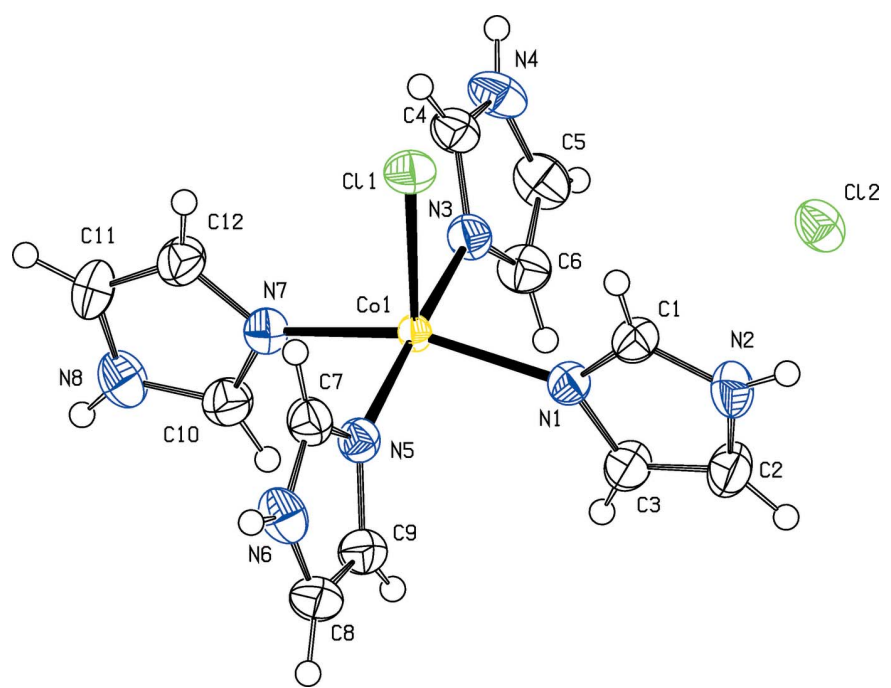

Figure 1

Unité asymétrique du composé [Co(imidazole $\left.)_{4} \mathrm{Cl}\right] \mathrm{Cl}$. Les éllipsoïdes ont été définis avec $50 \%$ de probabilité.

chlorure libre $\mathrm{Cl}^{-}$(Fig. 1). L'ion complexe $\left[\mathrm{Co}\left(\mathrm{C}_{3} \mathrm{H}_{4} \mathrm{~N}_{2}\right) \mathrm{Cl}\right]^{+}$ est formé par un cation $\mathrm{Co}^{2+}$ coordiné à quarte molécules imidazoles par des liaisons $\mathrm{Co}-\mathrm{N}$ et un seul anion chlorure (Co-Cl1). Ceci conduit à un environnement pyramide à base carrée (pentaèdre) autour du cation $\mathrm{Co}^{2+}$ (Fig. 1 et tableau 1$)$. Le détail de la structure montre que le polyèdre de coordination du cobalt $\mathrm{CoN}_{4} \mathrm{Cl}$ est une pyramide distordue. En effet, la distance $\mathrm{Co}-\mathrm{Cl}$ est de 2,615 (6) $\AA$ et les distances $\mathrm{Co}-\mathrm{N}$ varient de 1.999 (2) à 2.011 (2) $\AA$. En plus, les angles $\mathrm{Cl}-\mathrm{Co}-$ $\mathrm{N}$ ont des valeurs variables entre $104,28(7)$ et $92,40(8)^{\circ}$, les angles trans $\mathrm{N}-\mathrm{Co}-\mathrm{N}$ sont de 157,60 (9) et 174,57 (9) ${ }^{\circ}$ et les angles cis $\mathrm{N}-\mathrm{Co}-\mathrm{N}$ varie de 88,92 (11) à 90,26 (11) ${ }^{\circ}$. Les pentaèdres $\mathrm{CoN}_{4} \mathrm{Cl}$ sont isolés les uns des autres avec une distance minimale $\mathrm{Co}-\mathrm{Co}=7,3997$ (7) $\AA$. Cette distance est supérieure à celle qui permet de prévoir un couplage magnétique (Decaroli et al., 2015). Les groupements imida-



Figure 2

Projection de la structure du composé $\left[\mathrm{Co}(\text { imidazole })_{4} \mathrm{Cl}\right] \mathrm{Cl}$ dans le plan $b c$ montrant l'arrangement en couches.
Tableau 1

Paramètres géométriques sélectionnés $\left(\AA,^{\circ}\right)$.

\begin{tabular}{lrlr}
\hline Co1-N3 & $1,999(2)$ & Co1-N1 & $2,011(2)$ \\
Co1-N5 & $2,001(2)$ & Co1-Cl1 & $2,6151(7)$ \\
Co1-N7 & $2,003(2)$ & & \\
& & & \\
N3-Co1-N5 & $174,57(9)$ & N7-Co1-N1 & $157,60(9)$ \\
N3-Co1-N7 & $89,27(12)$ & N3-Co1-Cl1 & $93,01(9)$ \\
N5-Co1-N7 & $88,92(11)$ & N5-Co1-Cl1 & $92,40(8)$ \\
N3-Co1-N1 & $89,46(11)$ & N7-Co1-Cl1 & $104,28(7)$ \\
N5-Co1-N1 & $90,26(11)$ & N1-Co1-Cl1 & $98,13(7)$ \\
\hline
\end{tabular}

Tableau 2

Géométrie des liaisons hydrogènes $\left(\AA{ }^{\circ}{ }^{\circ}\right)$.

$\operatorname{Im} 3$ = centroïde du cycle imidazole N5/N6/C7-C9.

\begin{tabular}{lllll}
\hline$D-\mathrm{H} \cdots A$ & $D-\mathrm{H}$ & $\mathrm{H} \cdots A$ & $D \cdots A$ & $D-\mathrm{H} \cdots A$ \\
\hline $\mathrm{N} 2-\mathrm{H} 2 N \cdots \mathrm{Cl} 2$ & 0,86 & 2,53 & $3,180(3)$ & 133 \\
$\mathrm{~N} 4-\mathrm{H} 4 N \cdots \mathrm{Cl} 2^{\mathrm{i}}$ & 0,86 & 2,40 & $3,247(3)$ & 168 \\
$\mathrm{~N} 6-\mathrm{H} 6 N \cdots \mathrm{Cl} 2^{\mathrm{ii}}$ & 0,86 & 2,40 & $3,251(3)$ & 169 \\
$\mathrm{~N} 8-\mathrm{H} 8 N \cdots \mathrm{Cl} 1^{\mathrm{iii}}$ & 0,86 & 2,44 & $3,254(3)$ & 158 \\
$\mathrm{C} 3-\mathrm{H} 3 \cdots \mathrm{C} 2^{\mathrm{iii}}$ & 0,93 & 2,78 & $3,639(3)$ & 154 \\
$\mathrm{C} 4-\mathrm{H} 4 \cdots \mathrm{Cl} 1^{1 \mathrm{v}}$ & 0,93 & 2,78 & $3,541(4)$ & 140 \\
$\mathrm{C} 10-\mathrm{H} 10 \cdots \mathrm{Cl} 2^{\mathrm{iii}}$ & 0,93 & 2,77 & $3,657(3)$ & 160 \\
$\mathrm{C} 2-\mathrm{H} 2 \cdots \mathrm{Im} 3^{\mathrm{v}}$ & 0,93 & 2,82 & $3,526(3)$ & 134 \\
\hline
\end{tabular}

Codes de symétrie: (i) $x-\frac{1}{2},-y+\frac{1}{2}, z+\frac{1}{2}$; (ii) $x-\frac{1}{2},-y+\frac{1}{2}, z-\frac{1}{2}$; (iii) $x-1, y, z$; (iv) $-x+2,-y,-z+1 ;$ (v) $-x+\frac{3}{2}, y+\frac{1}{2},-z+\frac{1}{2}$.

zoles sont plans avec des déviations standards par rapport aux plans moyens inferieures à $0,3 \%$. Les distances $\mathrm{C}-\mathrm{N}$ varient de 1,314 (4) à 1,379 (4) $\AA$ et les distances $\mathrm{C}-\mathrm{C}$ varient de 1,334 (4) à 1,357 (4) A. Ces caractéristiques géométriques sont comparables à celles observées dans les composés analogues. Enfin, on note que la maille élémentaire du composé étudié présente un volume légèrement supérieur à celui du composé isoformulaire $\left[\mathrm{Cu}\left(\mathrm{C}_{3} \mathrm{H}_{4} \mathrm{~N}_{2}\right)_{4} \mathrm{Cl}\right] \mathrm{Cl}$, cet écart est de $1,3 \%$ et peut être expliqué par le rayon du cation $\mathrm{Co}^{2+}$ qui est supérieur à celui du cation $\mathrm{Cu}^{2+}\left[R\left(\mathrm{Co}^{2+}\right)=0,67 \AA\right.$ et $R\left(\mathrm{Cu}^{2+}\right)=$ 0,65 ̊; Shannon, 1976].

\section{Caractéristiques supramoléculaires}

La structure du composé peut être décrite comme une succession de couches parallèles au plan (010) et imbriquées les unes dans les autres (Fig. 2). Dans chacune des couches les ligands Cl1 permettent la connexion des groupements complexes en rubans infinis par des liaisons hydrogène N8H8N...Cl1 (Fig. 3 et tableau 2). Les anions $\mathrm{Cl}^{-}$se situent dans ces rubans et permettent la cohésion dans la couche. En effet chacun de ces anions établi trois liaisons hydrogène N2$\mathrm{H} 2 N \cdots \mathrm{Cl} 2, \mathrm{~N} 4-\mathrm{H} 4 N \cdots \mathrm{Cl} 2$ et $\mathrm{N} 6-\mathrm{H} 6 N \cdots \mathrm{Cl} 2$ engageant un cation complexe du même ruban et deux autres situés dans des rubans adjacents (Fig. 3 et table 2). En plus des liaisons hydrogène relativement fortes $\mathrm{N}-\mathrm{H} \cdots \mathrm{Cl}$, d'autres liaisons de type $\mathrm{C}-\mathrm{H} \cdots \mathrm{Cl}$, de plus faibles énergies contribuent à la cohésion dans les couches. En effet, l'anion Cl1 établi une liaison $\mathrm{C} 4-\mathrm{H} 4 \cdots \mathrm{Cl} 1$ engageant deux rubans adjacents alors que l'anion $\mathrm{Cl} 2$ établi deux liaisons $\mathrm{C} 3-\mathrm{H} 3 \cdots \mathrm{Cl} 2$ et $\mathrm{C} 10-$ $\mathrm{H} 10 \cdots \mathrm{Cl} 2$ appartenant à un même groupement complexe 


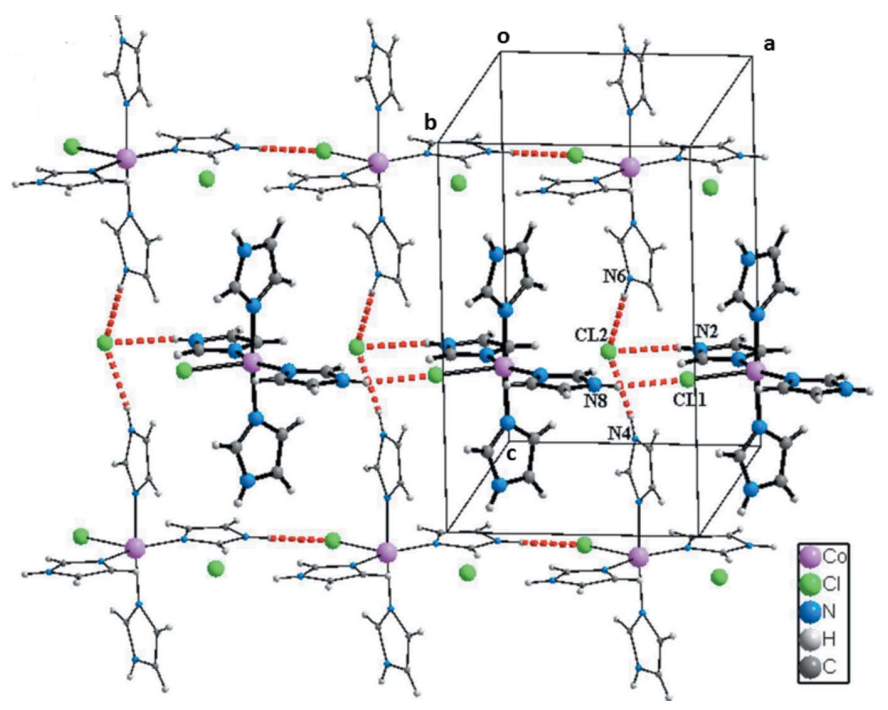

Figure 3

Cohésion dans une couche du composé [Co(imidazole $\left.)_{4} \mathrm{Cl}\right] \mathrm{Cl}$ par des liaisons hydrogène $\mathrm{N}-\mathrm{H} \cdots \mathrm{Cl}$.

adjacent du même ruban (Fig. 4 et tableau 2). La Fig. 5 (et tableau 2), montre que la connexion entre couches fait intervenir des liaisons hydrogène de faibles énergies $\mathrm{C} 4-\mathrm{H} 4 \cdots \mathrm{Cl} 1$ et $\mathrm{C} 2-\mathrm{H} 2 \cdots \operatorname{Im} 3$ en plus des interactions $\pi-\pi$ faisant intervenir les groupements imidazoles $\operatorname{Im} 1 \cdots \operatorname{Im} 4$ [3,914 (2) $\AA$ ], $\operatorname{Im} 4 \cdots \operatorname{Im} 4^{\mathrm{iii}}[3,794(2) \AA]$ et $\operatorname{Im} 4^{\mathrm{iii}} \cdots \operatorname{Im} 1^{\mathrm{ii}}[3,914$ (2) $\AA$ ] , avec Im = centroïde du cycle imidazole; Im1: N1/N2/C1-C3; Im4: N7/N8/C10-C12; pour les opérations de symétrie voir Fig. 5).

\section{Enquête de base de données}

L'étude structurale déjà effectuée (Morzyk-Ociepa et al., 2012) a mis en évidence le polymorphisme des complexes de

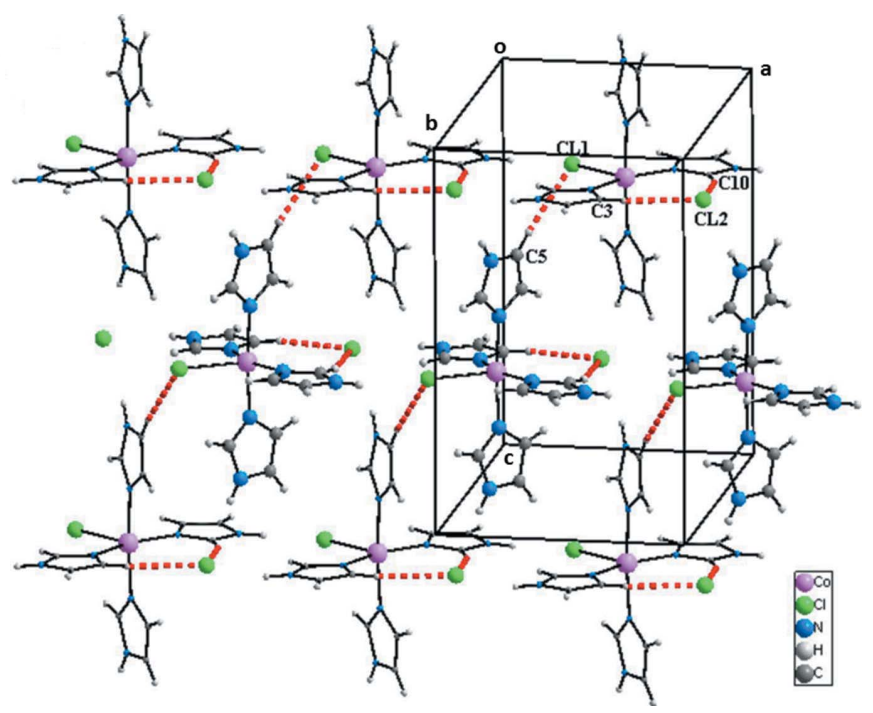

Figure 4

Cohésion dans une couche du composé $\left[\mathrm{Co}(\right.$ imidazole $\left.){ }_{4} \mathrm{Cl}\right] \mathrm{Cl}$ par des liaisons hydrogène $\mathrm{C}-\mathrm{H} \cdots \mathrm{Cl}$.



Figure 5

Liaisons entre couches dans la structure du composé [Co(imidazole) ${ }_{4} \mathrm{Cl}$ ] Cl. [Codes de symétrie: (i) $-x+3,-y+2,-z$; (ii) $-x+1,-y+2$, $-z+1$; (iii) $-x+1,-y+2,-z+1$.]

cuivre $\left[\mathrm{Cu}(\mathrm{Im})_{4} \mathrm{Cl}\right] \mathrm{Cl}$ en identifiant deux formes structurales qu'ils ont noté (1) et (2). Cette étude est la seule, à nôtre connaissance, qui a évoquée la présence de la forme (2) des composés $\left[M(\operatorname{Im}){ }_{4} X\right] X$ (avec $M=$ métal de transition et $X=$ $\mathrm{Cl}, \mathrm{Br}$ ). Cependant, la forme (1) à fait l'objet au moins de quatre études structurales relatives aux composés $\left[\mathrm{Cu}(\mathrm{Im})_{4} \mathrm{Cl}\right] \mathrm{Cl}$ (Morzyk-Ociepa et al., 2012; Li et al., 2007; Wu et al., 2013) et $\left[\mathrm{Cu}(\mathrm{Im})_{4} \mathrm{Br}\right] \mathrm{Br}$ (Hossaini Sadr et al., 2004). En plus, un examen bibliographique montre que six composés complexes analogues et de formulation générale $\left[M(R-\operatorname{Im})_{4} X\right] X$ (avec $M=$ métal de transition, $R=$ groupement alkyle et $X=\mathrm{Cl}, \mathrm{Br}$ ) sont identifiés (Godlewska et al., 2011a,b, 2012, 2013; Atria et al., 2003; Liu et al., 2007). Dans la totalité des composés étudiés déjà cités, le métal central est le cuivre. Le présent travail est consacré à l'étude du complexe du cobalt $\left[\mathrm{Co}(\mathrm{Im})_{4} \mathrm{Cl}\right] \mathrm{Cl}$, isotype à la forme (1) du complexe de cuivre analogue.

\section{Etude vibrationnelle}

Le spectre IR de ce composé a été enregistré dans le domaine de fréquences qui s'étend de 400 à $4000 \mathrm{~cm}^{-1}$ (Fig. 6). L'attribution des modes internes et externes est basée sur la comparaison avec les composés de la littérature et des composés similaires (Moryk-Ociepa et al., 2012; Wu et al., 2013). Les bandes très larges qui apparaissent entre $3310 \mathrm{~cm}^{-1}$ et $3120 \mathrm{~cm}^{-1}$ correspondent à la vibration de valence de groupement $\mathrm{N}-\mathrm{H}$, une série de bandes observée entre 2970 et $1975 \mathrm{~cm}^{-1}$ est due à la vibration d'élongation des groupements $\mathrm{C}-\mathrm{H}$. Les bandes de déformation de $\mathrm{N}-\mathrm{H}$ se manifeste entre $1625 \mathrm{~cm}^{-1}$ et $1437 \mathrm{~cm}^{-1}$ alors que les vibrations de valence de $\mathrm{C}-\mathrm{C}$ et $\mathrm{C}-\mathrm{N}$ sont observées entre $1625 \mathrm{~cm}^{-1}$ et $1078 \mathrm{~cm}^{-1}$. 


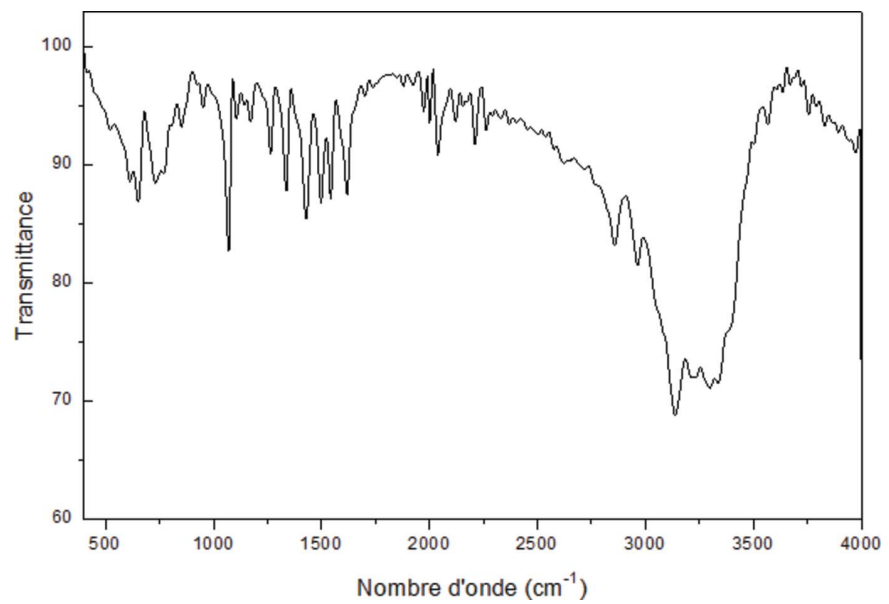

Figure 6

Spectre d'absorption infra rouge à la température ambiante du composé $\left[\mathrm{Co}(\text { imidazole })_{4} \mathrm{Cl}\right] \mathrm{Cl}$.

Les pics détectés vers $950 \mathrm{~cm}^{-1}$ et $847 \mathrm{~cm}^{-1}$ sont attribués à la déformation des cycles imidazole, alors que ceux observés vers 783 et $737 \mathrm{~cm}^{-1}$ sont assignés aux vibrations $\pi \mathrm{CH}$.

\section{Synthèse et cristallisation}

Le composé complexe de cobalt de formule [Co(imidazole $)_{4^{-}}$ $\mathrm{Cl}] \mathrm{Cl}$ n'a pu être obtenu en une seule étape à partir des précurseurs chlorure de cobalt, et imidazole. L'obtention de ces cristaux à nécessité deux étapes (Fig. 7). La première étape (I) consiste à préparer des cristaux de $\left[\mathrm{Co}\left(\mathrm{H}_{2} \mathrm{O}\right)_{4}\left(\mathrm{C}_{4} \mathrm{H}_{2} \mathrm{O}_{4}\right)\right]$ à partir d'un mélange équimolaire de chlorure de cobalt, d'acide fumarique et d'imidazole en solution aqueuse. La solution obtenue, abandonnée quelques jours à la température ambiante, donne après évaporation lente des cristaux rose transparents en forme de prisme. L'étude structurale sur monocristal à montré que cette phase est déjà étudiée (Zheng $\&$ Xie, 2004). Dans une deuxième étape (II), une solution est préparée par dissolution des cristaux de ce composé intermédiaire $\left[\mathrm{Co}\left(\mathrm{H}_{2} \mathrm{O}\right)_{4}\left(\mathrm{C}_{4} \mathrm{H}_{2} \mathrm{O}_{4}\right)\right]$ et d'imidazole dans de l'éthanol absolu. Le rapport stoechiométrique de ces précurseurs est 1:4, respectivement. La solution obtenue, donne


Figure 7

Schéma réactionnel des deux étapes de la préparation du composé $\left[\mathrm{Co}(\text { imidazole })_{4} \mathrm{Cl}\right] \mathrm{Cl}$.
Tableau 3

Détails expérimentaux.

\begin{tabular}{ll}
\hline Données crystallines & \\
Formule chimique & {$\left[\mathrm{CoCl}\left(\mathrm{C}_{3} \mathrm{H}_{4} \mathrm{~N}_{2}\right)_{4}\right] \mathrm{Cl}$} \\
$M_{\mathrm{r}}$ & 402,16 \\
Système cristallin, groupe d'espace & Monoclinique, $P 2_{1} / n$ \\
Température $(\mathrm{K})$ & 293 \\
$a, b, c(\AA)$ & $8,8665(3), 13,3043(6), 13,9154(5)$ \\
$\beta\left({ }^{\circ}\right)$ & $89,998(2)$ \\
$V\left(\AA^{3}\right)$ & $1641,50(11)$ \\
$Z$ & 4 \\
Type de rayonnement & Mo $K \alpha$ \\
$\mu\left(\mathrm{mm}^{-1}\right)$ & 1,38 \\
Taille des cristaux $(\mathrm{mm})$ & $0,22 \times 0,12 \times 0,10$ \\
& \\
Collection de données & Bruker SMART CCD area \\
Diffractomètre & detector \\
& Multi-scan $(S A D A B S ;$ Bruker, \\
Correction d'absorption & $1998)$ \\
& $0,840,0,882$ \\
$T_{\text {min }}, T_{\text {max }}$ & $2915,2915,2705$ \\
Nombre de réflexions mesurées, & \\
$\quad$ indépendantes et observées & \\
{$[I>2 \sigma(I)]$} & 0,023 \\
$R_{\text {int }}$ & 0,618 \\
$(\text { sin } \theta / \lambda)_{\text {max }}\left(\AA^{-1}\right)$ & \\
Affinement & $0,027,0,072,1,09$ \\
$R\left[F^{2}>2 \sigma\left(F^{2}\right)\right], w R\left(F^{2}\right), S$ & 2915 \\
Nombre de réflexions & 209 \\
Nombre de paramètres & H-atom paramètres contraints \\
Traitement des atome $\mathrm{H}$ & $0,40,-0,29$ \\
$\Delta \rho_{\text {max }}, \Delta \rho_{\text {min }}\left(\mathrm{e} \AA^{-3}\right)$ &
\end{tabular}

Programmes informatiques: SMART et SAINT (Bruker, 1998), SHELXS97 (Sheldrick, 2008), SHELXL2013 (Sheldrick, 2015), DIAMOND (Brandenburg \& Putz, 1999), WinGX (Farrugia, 2012) et PLATON (Spek, 2009).

après deux semaines d'évaporation lente et à la température ambiante, des cristaux bleu transparents en forme de prisme.

\section{Affinement}

Les données cristallines, les conditions de la collecte des intensités et les résultats de l'affinement final sont consignées dans le tableau 3. L'isotypie de ce composé à celui du cuivre à facilité l'attribution des pics. Malgré la localisation des différents groupements de la structure plusieurs anomalies ont été observées: facteurs de reliabilités élevés $(R=0,212$ et $R w=$ $0,516)$, pics résiduels intenses $\left[(\Delta \rho)_{\max }=6,05\right.$ e/ $/ \AA^{3}$ et $(\Delta \rho)_{\text {min }}$ $\left.=-2,40 \mathrm{e} / \AA^{3}\right]$ et $\mathrm{GOF}=7,80$. Ces anomalies sont interprétées comme signe de macle dans le cristal étudié. Cette macle est prévisible puisque la maille est monoclinique avec un paramètre $\beta \simeq 90^{\circ}$. L'utilisation de l'instruction TWIN mise en oeuvre par le logiciel SHELXL2013 (Sheldrick, 2015) à confirmé cette hypothèse avec un coefficient BASF = 0,431 (1). L'affinement de ce modèle structural à permis d'éliminer toutes les anomalies déjà citées $[R=0,027, R w=$ $0,072,(\Delta \rho)_{\max }=0,40 \mathrm{e} / \AA^{3},(\Delta \rho)_{\min }=-0,29 \mathrm{e} / \AA^{3}$ et $\mathrm{GOF}=$ $1,09]$. La localisation des atomes d'hydrogène est effectuée sur la base de considérations géométriques et affinés en imposant des contraintes à la distance et à l'agitation thermique: $\mathrm{C}-\mathrm{H}=$ $0,93 \AA, \mathrm{N}-\mathrm{H}=0,86 \AA$ avec $U_{\text {iso }}(\mathrm{H})=1,2 U_{\text {eq }}(\mathrm{C}, \mathrm{N})$. 


\section{Remerciements}

Nous remercions le Professeur Abdelhamid Ben Salah directeur du Laboratoire MESLAB, Département de chimie de la Faculté des Sciences de Sfax, Université de Sfax, pour la collecte des intensités de diffraction des rayons $\mathrm{X}$ sur monocristal.

\section{Références}

Atria, A. M., Cortés, P., Garland, M. T. \& Baggio, R. (2003). Acta Cryst. E59, m1075-m1077.

Bogle, R. G., Whitley, G. S., Soo, S. C., Johnstone, A. P. \& Vallance, P. (1994). Br. J. Pharmacol. 111, 1257-1261.

Brandenburg, K. \& Putz, H. (1999). DIAMOND. Crystal Impact GbR, Bonn, Allemagne.

Bruker (1998). SMART, SAINT et SADABS. Bruker AXS Inc., Madison, Wisconsin, EU.

Castaño, T., Encinas, A., Pérez, C., Castro, A., Campillo, N. E. \& Gil, C. (2008). Bioorg. Med. Chem. 16, 6193-6206.

Decaroli, C., Arevalo-Lopez, A. M., Woodall, C. H., Rodriguez, E. E., Attfield, J. P., Parker, S. F. \& Stock, C. (2015). Acta Cryst. B71, 2024.

Farrugia, L. J. (2012). J. Appl. Cryst. 45, 849-854.

Godlewska, S., Baranowska, K., Socha, J. \& Dolega, A. (2011a). Acta Cryst. E67, m1906.
Godlewska, S., Jezierska, J., Baranowska, K., Augustin, E. \& Dołęga, A. (2013). Polyhedron, 65, 288-297.

Godlewska, S., Kelm, H., Krüger, H.-J. \& Dołęga, A. (2012). Acta Cryst. E68, m1529.

Godlewska, S., Socha, J., Baranowska, K. \& Dołęga, A. (2011b). Acta Cryst. E67, m1338.

Hossaini Sadr, M., Zare, D., Lewis, W., Wikaira, J., Robinson, W. T. \& Ng, S. W. (2004). Acta Cryst. E60, m1324-m1326.

Li, T. B., Hu, Y. L., Li, J. K. \& He, G. F. (2007). Acta Cryst. E63, $\mathrm{m} 2536$.

Liu, F.-Q., Liu, W.-L., Li, W., Li, R.-X. \& Liu, G.-Y. (2007). Acta Cryst. E63, m2454.

Morzyk-Ociepa, B., Różycka-Sokołowska, E. \& Michalska, D. (2012). J. Mol. Struct. 1028, 49-56.

OECD (2003). Screening Information Dataset Imidazole. CAS No. 288-32-4. UNEP Publications.

Shannon, R. D. (1976). Acta Cryst. A32, 751-767.

Shargel, L., Mutnick, A. H., Souney, P. F. \& Swanson, L. N. (2006). Dans Comprehensive Pharmacy Review, 6eme ed. Philadelphia: Lippincott Williams \& Wilkins.

Sheldrick, G. M. (2008). Acta Cryst. A64, 112-122.

Sheldrick, G. M. (2015). Acta Cryst. C71, 3-8.

Spek, A. L. (2009). Acta Cryst. D65, 148-155.

Wu, B. D., Zhou, Z. N., Bi, Y. G., Yang, L., Zhang, J. G. \& Zhang, T. L. (2013). Z. Anorg. Allg. Chem. 639, 799-803.

Zheng, Y. Q. \& Xie, H. Z. (2004). J. Solid State Chem. 177, 1352-1358. 


\section{supporting information}

Acta Cryst. (2015). E71, 1185-1189 [doi:10.1107/S2056989015015807]

\section{Etude structurale et vibrationnelle d'un nouveau composé complexe de cobalt: $\left[\mathrm{Co}(\text { imidazole })_{4} \mathrm{Cl}\right] \mathrm{Cl}$}

\section{Amira Derbel, Tahar Mhiri et Mohsen Graia}

\section{Computing details}

Data collection: SMART (Bruker, 1998); cell refinement: SAINT (Bruker, 1998); data reduction: SAINT (Bruker, 1998); program(s) used to solve structure: SHELXS97 (Sheldrick, 2008); program(s) used to refine structure: SHELXL2013 (Sheldrick, 2015); molecular graphics: DIAMOND (Brandenburg \& Putz, 1999) et PLATON (Spek, 2009); software used to prepare material for publication: $\operatorname{Win} G X$ (Farrugia, 2012) et PLATON (Spek, 2009).

\section{Chloridotetrakis $\left(1 H\right.$-imidazole- $\left.\kappa N^{3}\right)$ cobalt(II) chloride}

\section{Crystal data}

$\left[\mathrm{CoCl}\left(\mathrm{C}_{3} \mathrm{H}_{4} \mathrm{~N}_{2}\right)_{4}\right] \mathrm{Cl}$

$M_{r}=402.16$

Monoclinic, $P 2{ }_{1} / n$

$a=8.8665(3) \AA$

$b=13.3043(6) \AA$

$c=13.9154(5) \AA$

$\beta=89.998(2)^{\circ}$

$V=1641.50(11) \AA^{3}$

\section{Data collection}

Bruker SMART CCD area-detector diffractometer

$\varphi$ and $\omega$ scans

Absorption correction: multi-scan (SADABS; Bruker, 1998)

$T_{\min }=0.840, T_{\max }=0.882$

2915 measured reflections

\section{Refinement}

Refinement on $F^{2}$

Least-squares matrix: full

$R\left[F^{2}>2 \sigma\left(F^{2}\right)\right]=0.027$

$w R\left(F^{2}\right)=0.072$

$S=1.09$

2915 reflections

209 parameters

0 restraints

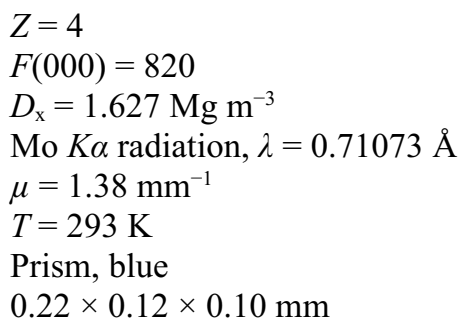

2915 independent reflections

2705 reflections with $I>2 \sigma(I)$

$R_{\text {int }}=0.023$

$\theta_{\max }=26.1^{\circ}, \theta_{\min }=2.1^{\circ}$

$h=-10 \rightarrow 10$

$k=-16 \rightarrow 16$

$l=0 \rightarrow 16$

Hydrogen site location: inferred from neighbouring sites

$\mathrm{H}$-atom parameters constrained

$w=1 /\left[\sigma^{2}\left(F_{\mathrm{o}}{ }^{2}\right)+(0.040 P)^{2}+0.4321 P\right]$

where $P=\left(F_{\mathrm{o}}{ }^{2}+2 F_{\mathrm{c}}{ }^{2}\right) / 3$

$(\Delta / \sigma)_{\max }<0.001$

$\Delta \rho_{\max }=0.40 \mathrm{e}^{-3}$

$\Delta \rho_{\min }=-0.29$ e $\AA^{-3}$ 


\section{Special details}

Geometry. All e.s.d.'s (except the e.s.d. in the dihedral angle between two 1.s. planes) are estimated using the full covariance matrix. The cell e.s.d.'s are taken into account individually in the estimation of e.s.d.'s in distances, angles and torsion angles; correlations between e.s.d.'s in cell parameters are only used when they are defined by crystal symmetry. An approximate (isotropic) treatment of cell e.s.d.'s is used for estimating e.s.d.'s involving 1.s. planes.

Refinement. Refined as a 2-component twin.

Fractional atomic coordinates and isotropic or equivalent isotropic displacement parameters $\left(\hat{A}^{2}\right)$

\begin{tabular}{|c|c|c|c|c|}
\hline & $x$ & $y$ & $z$ & $U_{\text {iso }} * / U_{\text {eq }}$ \\
\hline Co1 & $0.81347(4)$ & $0.21324(2)$ & 0.37752 & $0.01963(9)$ \\
\hline $\mathrm{Cl1}$ & $1.05621(8)$ & $0.10160(5)$ & $0.37941(7)$ & $0.03569(16)$ \\
\hline $\mathrm{Cl} 2$ & $1.44553(8)$ & $0.40796(6)$ & $0.37507(7)$ & $0.03951(17)$ \\
\hline N1 & $0.9146(3)$ & $0.34849(16)$ & $0.3746(2)$ & $0.0300(5)$ \\
\hline $\mathrm{C} 1$ & 1.0619 & $0.3624(2)$ & $0.3717(3)$ & $0.0328(6)$ \\
\hline H1 & 1.1328 & 0.3109 & 0.3701 & $0.039 *$ \\
\hline N2 & 1.0955 (3) & $0.45971(18)$ & $0.3714(2)$ & $0.0401(6)$ \\
\hline $\mathrm{H} 2 \mathrm{~N}$ & 1.1848 & 0.4849 & 0.3702 & $0.048 *$ \\
\hline $\mathrm{C} 2$ & $0.9645(3)$ & $0.5127(2)$ & $0.3734(3)$ & $0.0435(7)$ \\
\hline $\mathrm{H} 2$ & 0.9539 & 0.5822 & 0.3737 & $0.052 *$ \\
\hline C3 & 0.8525 & $0.4432(2)$ & $0.3748(3)$ & $0.0385(7)$ \\
\hline $\mathrm{H} 3$ & 0.7498 & 0.4575 & 0.3757 & $0.046^{*}$ \\
\hline N3 & $0.8045(4)$ & $0.22115(18)$ & $0.52089(16)$ & $0.0319(6)$ \\
\hline $\mathrm{C} 4$ & $0.8591(4)$ & 0.1555 & 0.5825 & $0.0344(9)$ \\
\hline H4 & 0.9140 & 0.0985 & 0.5658 & $0.041 *$ \\
\hline N4 & $0.8246(4)$ & $0.1822(2)$ & $0.67242(18)$ & $0.0415(7)$ \\
\hline $\mathrm{H} 4 \mathrm{~N}$ & 0.8488 & 0.1499 & 0.7237 & $0.050 *$ \\
\hline $\mathrm{C} 5$ & $0.7453(5)$ & $0.2690(3)$ & $0.6690(2)$ & $0.0443(10)$ \\
\hline H5 & 0.7074 & 0.3049 & 0.7210 & $0.053 *$ \\
\hline C6 & $0.7323(5)$ & 0.2928 & $0.5757(2)$ & $0.0385(9)$ \\
\hline H6 & 0.6823 & 0.3489 & 0.5518 & $0.046^{*}$ \\
\hline N5 & 0.8038 & $0.21234(18)$ & $0.23384(16)$ & $0.0289(5)$ \\
\hline $\mathrm{C} 7$ & 0.8709 (4) & 0.1515 & $0.1730(2)$ & $0.0357(9)$ \\
\hline $\mathrm{H} 7$ & 0.9358 & 0.0996 & 0.1903 & $0.043 *$ \\
\hline N6 & $0.8326(4)$ & $0.1746(2)$ & 0.08285 (18) & $0.0391(7)$ \\
\hline $\mathrm{H} 6 \mathrm{~N}$ & 0.8641 & 0.1450 & 0.0317 & $0.047^{*}$ \\
\hline $\mathrm{C} 8$ & $0.7344(5)$ & $0.2537(3)$ & $0.0865(2)$ & $0.0417(9)$ \\
\hline H8 & 0.6885 & 0.2855 & 0.0346 & $0.050 *$ \\
\hline $\mathrm{C} 9$ & $0.7177(5)$ & $0.2762(2)$ & 0.1793 (2) & $0.0363(9)$ \\
\hline H9 & 0.6569 & 0.3273 & 0.2033 & $0.044 *$ \\
\hline N7 & $0.6433(3)$ & $0.11427(17)$ & $0.3800(2)$ & $0.0308(5)$ \\
\hline $\mathrm{C} 10$ & 0.4975 & $0.1353(2)$ & 0.3745 & $0.0391(7)$ \\
\hline H10 & 0.4577 & 0.1999 & 0.3716 & $0.047 *$ \\
\hline N8 & 0.4150 & 0.05047 (19) & $0.3737(3)$ & $0.0429(6)$ \\
\hline $\mathrm{H} 8 \mathrm{~N}$ & 0.3184 & 0.0467 & 0.3702 & $0.051 *$ \\
\hline C11 & $0.5122(3)$ & $-0.0281(2)$ & $0.3794(3)$ & $0.0444(8)$ \\
\hline H11 & 0.4874 & -0.0960 & 0.3805 & $0.053 *$ \\
\hline C12 & $0.6517(3)$ & $0.0117(2)$ & $0.3829(3)$ & $0.0396(7)$ \\
\hline
\end{tabular}


supporting information



Geometric parameters $\left(\AA,{ }^{\circ}\right)$

\begin{tabular}{llll}
\hline Co1-N3 & $1.999(2)$ & $\mathrm{C} 5-\mathrm{H} 5$ & 0.9300 \\
Co1-N5 & $2.001(2)$ & $\mathrm{C} 6-\mathrm{H} 6$ & 0.9300 \\
$\mathrm{Co} 1-\mathrm{N} 7$ & $2.003(2)$ & $\mathrm{N} 5-\mathrm{C} 7$ & $1.314(4)$ \\
$\mathrm{Co} 1-\mathrm{N} 1$ & $2.011(2)$ & $\mathrm{N} 5-\mathrm{C} 9$ & $1.372(4)$ \\
$\mathrm{Co} 1-\mathrm{C} 11$ & $2.6151(7)$ & $\mathrm{C} 7-\mathrm{N} 6$ & $1.336(4)$ \\
$\mathrm{N} 1-\mathrm{C} 1$ & $1.319(4)$ & $\mathrm{C} 7-\mathrm{H} 7$ & 0.9300 \\
$\mathrm{~N} 1-\mathrm{C} 3$ & $1.375(3)$ & $\mathrm{N} 6-\mathrm{C} 8$ & $1.367(5)$ \\
$\mathrm{C} 1-\mathrm{N} 2$ & $1.328(4)$ & $\mathrm{N} 6-\mathrm{H} 6 \mathrm{~N}$ & 0.8600 \\
$\mathrm{C} 1-\mathrm{H} 1$ & 0.9300 & $\mathrm{C} 8-\mathrm{C} 9$ & $1.334(4)$ \\
$\mathrm{N} 2-\mathrm{C} 2$ & $1.358(4)$ & $\mathrm{C} 8-\mathrm{H} 8$ & 0.9300 \\
$\mathrm{~N} 2-\mathrm{H} 2 \mathrm{~N}$ & 0.8600 & $\mathrm{C} 9-\mathrm{H} 9$ & 0.9300 \\
$\mathrm{C} 2-\mathrm{C} 3$ & $1.357(4)$ & $\mathrm{N} 7-\mathrm{C} 10$ & $1.324(4)$ \\
$\mathrm{C} 2-\mathrm{H} 2$ & 0.9300 & $\mathrm{~N} 7-\mathrm{C} 12$ & $1.368(4)$ \\
$\mathrm{C} 3-\mathrm{H} 3$ & 0.9300 & $\mathrm{C} 10-\mathrm{N} 8$ & $1.345(4)$ \\
$\mathrm{N} 3-\mathrm{C} 4$ & $1.316(4)$ & $\mathrm{C} 10-\mathrm{H} 10$ & 0.9300 \\
$\mathrm{~N} 3-\mathrm{C} 6$ & $1.379(4)$ & $\mathrm{N} 8-\mathrm{C} 11$ & $1.357(4)$ \\
$\mathrm{C} 4-\mathrm{N} 4$ & $1.336(4)$ & $\mathrm{N} 8-\mathrm{H} 8 \mathrm{~N}$ & 0.8600
\end{tabular}




\begin{tabular}{|c|c|c|c|}
\hline $\mathrm{C} 4-\mathrm{H} 4$ & 0.9300 & $\mathrm{C} 11-\mathrm{C} 12$ & $1.346(4)$ \\
\hline $\mathrm{N} 4-\mathrm{C} 5$ & $1.352(5)$ & $\mathrm{C} 11-\mathrm{H} 11$ & 0.9300 \\
\hline $\mathrm{N} 4-\mathrm{H} 4 \mathrm{~N}$ & 0.8600 & $\mathrm{C} 12-\mathrm{H} 12$ & 0.9300 \\
\hline $\mathrm{C} 5-\mathrm{C} 6$ & $1.341(5)$ & & \\
\hline $\mathrm{N} 3-\mathrm{Co} 1-\mathrm{N} 5$ & $174.57(9)$ & $\mathrm{C} 6-\mathrm{C} 5-\mathrm{H} 5$ & 126.9 \\
\hline $\mathrm{N} 3-\mathrm{Co} 1-\mathrm{N} 7$ & $89.27(12)$ & $\mathrm{N} 4-\mathrm{C} 5-\mathrm{H} 5$ & 126.9 \\
\hline N5-Co1-N7 & $88.92(11)$ & $\mathrm{C} 5-\mathrm{C} 6-\mathrm{N} 3$ & $109.4(3)$ \\
\hline $\mathrm{N} 3-\mathrm{Co} 1-\mathrm{N} 1$ & $89.46(11)$ & $\mathrm{C} 5-\mathrm{C} 6-\mathrm{H} 6$ & 125.3 \\
\hline $\mathrm{N} 5-\mathrm{Co} 1-\mathrm{N} 1$ & $90.26(11)$ & $\mathrm{N} 3-\mathrm{C} 6-\mathrm{H} 6$ & 125.3 \\
\hline $\mathrm{N} 7-\mathrm{Co} 1-\mathrm{N} 1$ & $157.60(9)$ & $\mathrm{C} 7-\mathrm{N} 5-\mathrm{C} 9$ & $106.1(3)$ \\
\hline $\mathrm{N} 3-\mathrm{Co} 1-\mathrm{Cl}$ & $93.01(9)$ & $\mathrm{C} 7-\mathrm{N} 5-\mathrm{Co} 1$ & $128.9(2)$ \\
\hline $\mathrm{N} 5-\mathrm{Co} 1-\mathrm{Cl}$ & $92.40(8)$ & $\mathrm{C} 9-\mathrm{N} 5-\mathrm{Co} 1$ & $125.0(2)$ \\
\hline $\mathrm{N} 7-\mathrm{Co} 1-\mathrm{Cl}$ & $104.28(7)$ & $\mathrm{N} 5-\mathrm{C} 7-\mathrm{N} 6$ & $110.4(3)$ \\
\hline $\mathrm{N} 1-\mathrm{Co} 1-\mathrm{Cl}$ & $98.13(7)$ & $\mathrm{N} 5-\mathrm{C} 7-\mathrm{H} 7$ & 124.8 \\
\hline $\mathrm{C} 1-\mathrm{N} 1-\mathrm{C} 3$ & $105.5(2)$ & $\mathrm{N} 6-\mathrm{C} 7-\mathrm{H} 7$ & 124.8 \\
\hline $\mathrm{C} 1-\mathrm{N} 1-\mathrm{Co} 1$ & $124.59(18)$ & $\mathrm{C} 7-\mathrm{N} 6-\mathrm{C} 8$ & $107.8(3)$ \\
\hline $\mathrm{C} 3-\mathrm{N} 1-\mathrm{Co} 1$ & $129.90(19)$ & $\mathrm{C} 7-\mathrm{N} 6-\mathrm{H} 6 \mathrm{~N}$ & 126.1 \\
\hline $\mathrm{N} 1-\mathrm{C} 1-\mathrm{N} 2$ & $111.0(3)$ & $\mathrm{C} 8-\mathrm{N} 6-\mathrm{H} 6 \mathrm{~N}$ & 126.1 \\
\hline $\mathrm{N} 1-\mathrm{C} 1-\mathrm{H} 1$ & 124.5 & $\mathrm{C} 9-\mathrm{C} 8-\mathrm{N} 6$ & $106.2(3)$ \\
\hline $\mathrm{N} 2-\mathrm{C} 1-\mathrm{H} 1$ & 124.5 & $\mathrm{C} 9-\mathrm{C} 8-\mathrm{H} 8$ & 126.9 \\
\hline $\mathrm{C} 1-\mathrm{N} 2-\mathrm{C} 2$ & $108.3(2)$ & $\mathrm{N} 6-\mathrm{C} 8-\mathrm{H} 8$ & 126.9 \\
\hline $\mathrm{C} 1-\mathrm{N} 2-\mathrm{H} 2 \mathrm{~N}$ & 125.8 & $\mathrm{C} 8-\mathrm{C} 9-\mathrm{N} 5$ & $109.6(3)$ \\
\hline $\mathrm{C} 2-\mathrm{N} 2-\mathrm{H} 2 \mathrm{~N}$ & 125.8 & $\mathrm{C} 8-\mathrm{C} 9-\mathrm{H} 9$ & 125.2 \\
\hline $\mathrm{C} 3-\mathrm{C} 2-\mathrm{N} 2$ & $105.8(2)$ & $\mathrm{N} 5-\mathrm{C} 9-\mathrm{H} 9$ & 125.2 \\
\hline $\mathrm{C} 3-\mathrm{C} 2-\mathrm{H} 2$ & 127.1 & $\mathrm{C} 10-\mathrm{N} 7-\mathrm{C} 12$ & $105.4(3)$ \\
\hline $\mathrm{N} 2-\mathrm{C} 2-\mathrm{H} 2$ & 127.1 & $\mathrm{C} 10-\mathrm{N} 7-\mathrm{Co} 1$ & $126.5(2)$ \\
\hline $\mathrm{C} 2-\mathrm{C} 3-\mathrm{N} 1$ & $109.3(3)$ & $\mathrm{C} 12-\mathrm{N} 7-\mathrm{Co} 1$ & $128.0(2)$ \\
\hline $\mathrm{C} 2-\mathrm{C} 3-\mathrm{H} 3$ & 125.3 & $\mathrm{~N} 7-\mathrm{C} 10-\mathrm{N} 8$ & $110.7(3)$ \\
\hline $\mathrm{N} 1-\mathrm{C} 3-\mathrm{H} 3$ & 125.3 & $\mathrm{~N} 7-\mathrm{C} 10-\mathrm{H} 10$ & 124.6 \\
\hline $\mathrm{C} 4-\mathrm{N} 3-\mathrm{C} 6$ & $105.6(3)$ & $\mathrm{N} 8-\mathrm{C} 10-\mathrm{H} 10$ & 124.6 \\
\hline $\mathrm{C} 4-\mathrm{N} 3-\mathrm{Co} 1$ & $126.9(2)$ & $\mathrm{C} 10-\mathrm{N} 8-\mathrm{C} 11$ & $107.5(2)$ \\
\hline $\mathrm{C} 6-\mathrm{N} 3-\mathrm{Co} 1$ & $127.4(2)$ & $\mathrm{C} 10-\mathrm{N} 8-\mathrm{H} 8 \mathrm{~N}$ & 126.2 \\
\hline $\mathrm{N} 3-\mathrm{C} 4-\mathrm{N} 4$ & $110.4(3)$ & $\mathrm{C} 11-\mathrm{N} 8-\mathrm{H} 8 \mathrm{~N}$ & 126.2 \\
\hline $\mathrm{N} 3-\mathrm{C} 4-\mathrm{H} 4$ & 124.8 & $\mathrm{C} 12-\mathrm{C} 11-\mathrm{N} 8$ & $106.4(3)$ \\
\hline $\mathrm{N} 4-\mathrm{C} 4-\mathrm{H} 4$ & 124.8 & $\mathrm{C} 12-\mathrm{C} 11-\mathrm{H} 11$ & 126.8 \\
\hline $\mathrm{C} 4-\mathrm{N} 4-\mathrm{C} 5$ & $108.3(3)$ & $\mathrm{N} 8-\mathrm{C} 11-\mathrm{H} 11$ & 126.8 \\
\hline $\mathrm{C} 4-\mathrm{N} 4-\mathrm{H} 4 \mathrm{~N}$ & 125.9 & $\mathrm{C} 11-\mathrm{C} 12-\mathrm{N} 7$ & $109.9(3)$ \\
\hline $\mathrm{C} 5-\mathrm{N} 4-\mathrm{H} 4 \mathrm{~N}$ & 125.9 & $\mathrm{C} 11-\mathrm{C} 12-\mathrm{H} 12$ & 125.0 \\
\hline $\mathrm{C} 6-\mathrm{C} 5-\mathrm{N} 4$ & $106.3(3)$ & $\mathrm{N} 7-\mathrm{C} 12-\mathrm{H} 12$ & 125.0 \\
\hline $\mathrm{C} 3-\mathrm{N} 1-\mathrm{C} 1-\mathrm{N} 2$ & $0.9(5)$ & $\mathrm{C} 9-\mathrm{N} 5-\mathrm{C} 7-\mathrm{N} 6$ & $0.7(4)$ \\
\hline $\mathrm{Co} 1-\mathrm{N} 1-\mathrm{C} 1-\mathrm{N} 2$ & $-179.3(2)$ & $\mathrm{Co} 1-\mathrm{N} 5-\mathrm{C} 7-\mathrm{N} 6$ & $178.6(2)$ \\
\hline $\mathrm{N} 1-\mathrm{C} 1-\mathrm{N} 2-\mathrm{C} 2$ & $-0.5(5)$ & $\mathrm{N} 5-\mathrm{C} 7-\mathrm{N} 6-\mathrm{C} 8$ & $-0.7(5)$ \\
\hline $\mathrm{C} 1-\mathrm{N} 2-\mathrm{C} 2-\mathrm{C} 3$ & $-0.1(5)$ & $\mathrm{C} 7-\mathrm{N} 6-\mathrm{C} 8-\mathrm{C} 9$ & $0.4(5)$ \\
\hline $\mathrm{N} 2-\mathrm{C} 2-\mathrm{C} 3-\mathrm{N} 1$ & $0.6(5)$ & $\mathrm{N} 6-\mathrm{C} 8-\mathrm{C} 9-\mathrm{N} 5$ & $0.0(5)$ \\
\hline $\mathrm{C} 1-\mathrm{N} 1-\mathrm{C} 3-\mathrm{C} 2$ & $-0.9(5)$ & $\mathrm{C} 7-\mathrm{N} 5-\mathrm{C} 9-\mathrm{C} 8$ & $-0.4(5)$ \\
\hline $\mathrm{Co} 1-\mathrm{N} 1-\mathrm{C} 3-\mathrm{C} 2$ & $179.3(3)$ & $\mathrm{Co} 1-\mathrm{N} 5-\mathrm{C} 9-\mathrm{C} 8$ & $-178.4(3)$ \\
\hline
\end{tabular}




$\begin{array}{llll}\mathrm{C} 6-\mathrm{N} 3-\mathrm{C} 4-\mathrm{N} 4 & 0.1(4) & \mathrm{C} 12-\mathrm{N} 7-\mathrm{C} 10-\mathrm{N} 8 & -0.1(5) \\ \mathrm{Co} 1-\mathrm{N} 3-\mathrm{C} 4-\mathrm{N} 4 & -176.3(3) & \mathrm{C} 1-\mathrm{N} 7-\mathrm{C} 10-\mathrm{N} 8 & 177.0(2) \\ \mathrm{N} 3-\mathrm{C} 4-\mathrm{N} 4-\mathrm{C} 5 & -0.4(5) & \mathrm{N} 7-\mathrm{C} 10-\mathrm{N} 8-\mathrm{C} 11 & 0.3(5) \\ \mathrm{C} 4-\mathrm{N} 4-\mathrm{C} 5-\mathrm{C} 6 & 0.5(5) & \mathrm{C} 10-\mathrm{N} 8-\mathrm{C} 11-\mathrm{C} 12 & -0.4(5) \\ \mathrm{N} 4-\mathrm{C} 5-\mathrm{C} 6-\mathrm{N} 3 & -0.4(5) & \mathrm{N} 8-\mathrm{C} 11-\mathrm{C} 12-\mathrm{N} 7 & 0.3(5) \\ \mathrm{C} 4-\mathrm{N} 3-\mathrm{C} 6-\mathrm{C} 5 & 0.2(5) & \mathrm{C} 10-\mathrm{N} 7-\mathrm{C} 12-\mathrm{C} 11 & -0.1(5) \\ \mathrm{C} 1-\mathrm{N} 3-\mathrm{C} 6-\mathrm{C} 5 & 176.6(3) & \mathrm{C} 1-\mathrm{N} 7-\mathrm{C} 12-\mathrm{C} 11 & -177.2(3)\end{array}$

Hydrogen-bond geometry $\left(\AA,{ }^{\circ}\right)$

$\operatorname{Im} 3=$ centroïde du cycle imidazole N5/N6/C7-C9

\begin{tabular}{lllll}
\hline$D-\mathrm{H} \cdots A$ & $D-\mathrm{H}$ & $\mathrm{H} \cdots A$ & $D \cdots A$ & $D-\mathrm{H} \cdots A$ \\
\hline $\mathrm{N} 2-\mathrm{H} 2 N \cdots \mathrm{Cl} 2$ & 0.86 & 2.53 & $3.180(3)$ & 133 \\
$\mathrm{~N} 4-\mathrm{H} 4 N \cdots \mathrm{Cl} 2^{\mathrm{i}}$ & 0.86 & 2.40 & $3.247(3)$ & 168 \\
$\mathrm{~N} 6-\mathrm{H} 6 N \cdots \mathrm{Cl} 2^{\mathrm{ii}}$ & 0.86 & 2.40 & $3.251(3)$ & 169 \\
$\mathrm{~N} 8-\mathrm{H} 8 N \cdots \mathrm{Cl} 1^{\mathrm{iii}}$ & 0.86 & 2.44 & $3.254(3)$ & 158 \\
$\mathrm{C} 3-\mathrm{H} 3 \cdots \mathrm{Cl} 2^{\mathrm{iii}}$ & 0.93 & 2.78 & $3.639(3)$ & 154 \\
$\mathrm{C} 4-\mathrm{H} 4 \cdots \mathrm{Cl} 1^{\mathrm{iv}}$ & 0.93 & 2.78 & $3.541(4)$ & 140 \\
$\mathrm{C} 10-\mathrm{H} 10 \cdots \mathrm{Cl} 2^{\mathrm{iii}}$ & 0.93 & 2.77 & $3.657(3)$ & 160 \\
$\mathrm{C} 2-\mathrm{H} 2 \cdots \mathrm{Im} 3^{\mathrm{v}}$ & 0.93 & 2.82 & $3.526(3)$ & 134
\end{tabular}

Symmetry codes: (i) $x-1 / 2,-y+1 / 2, z+1 / 2$; (ii) $x-1 / 2,-y+1 / 2, z-1 / 2$; (iii) $x-1, y, z$; (iv) $-x+2,-y,-z+1$; (v) $-x+3 / 2, y+1 / 2,-z+1 / 2$. 